\title{
ADVANCED METHODS FOR SIMULATION OUTPUT ANALYSIS
}

\author{
Christos Alexopoulos \\ School of Industrial and Systems Engineering \\ Georgia Institute of Technology \\ Atlanta, Georgia 30332-0205, U.S.A.
}

\author{
Andrew F. Seila \\ Terry College of Business \\ University of Georgia \\ Athens, GA 30602-6255, U.S.A.
}

\begin{abstract}
This paper reviews statistical methods for analyzing output data from computer simulations of single systems. In particular, it focuses on the problems of choosing initial conditions and estimating steady-state system parameters. The estimation techniques include the replication/deletion approach, the regenerative method, the batch means method, and the standardized time series method.
\end{abstract}

\section{INTRODUCTION}

A simulation study consists of several steps such as data collection, coding and verification, model validation, experimental design, output data analysis, and implementation. This paper focuses on statistical methods for computing confidence intervals for system performance measures from output data.

The primary purpose of most simulation studies is the approximation of prescribed system parameters with the objective of identifying parameter values that optimize some system performance measures. If some of the input processes driving a simulation are random, then the output data are also random and runs of the simulation program can only produce estimates of system performance measures. Unfortunately, a simulation run does not usually produce independent, identically distributed (i.i.d.) observations; therefore "classical" statistical techniques are not directly applicable to the analysis of simulation output.

There are two types of simulations with regard to output analysis:

1. Finite-horizon simulations: In this case the simulation starts in a specific state, such as the empty and idle state, and is run until some terminating event occurs. The output process is not expected to achieve any steady-state behavior and the value of any parameter estimated from the output data will depend upon the initial conditions. An example is the simulation of a building evacuation.
2. Steady-state simulations: The purpose of a steadystate simulation is the study of the long-run behavior of the system of interest. A performance measure of a system is called a steady-state parameter if it is a characteristic of the equilibrium distribution of an output stochastic process. The value of a steady-state parameter does not depend upon the initial conditions. An example is the simulation of a continuously operating communication system where the objective is the computation of the mean delay of a data packet.

Section 2 discusses methods for analyzing output from finite-horizon simulations. Section 3 reviews approaches for removing bias due to initial conditions in steady-state simulations. Section 4 presents techniques for point and interval estimation of steady-state parameters.

\section{FINITE-HORIZON SIMULATIONS}

Suppose that we simulate a system until $n$ output data $X_{1}, X_{2}, \ldots, X_{n}$ are collected with the objective of estimating $\mu=\mathrm{E}\left(\bar{X}_{n}\right)$, where $\bar{X}_{n}=\frac{1}{n} \sum_{i=1}^{n} X_{i}$ is the sample mean of the data. For example, $X_{i}$ may be the

transit time of unit $i$ through a network of queues or the total time station $i$ is busy during the $i$ th hour. Clearly, $\bar{X}_{n}$ is an unbiased estimator for $\mu$. Unfortunately, the $X_{i}$ 's are generally dependent random variables, making the estimation of the variance $\operatorname{Var}\left(\bar{X}_{n}\right)$ a nontrivial problem. In many queueing systems the $X_{i}$ 's are positively correlated. This causes the familiar estimator $S^{2}(n) / n=\sum_{i=1}^{n}\left(X_{i}-\bar{X}_{n}\right)^{2} /[n(n-1)]$ to be a highly biased estimator of $\operatorname{Var}\left(\bar{X}_{n}\right)$.

To overcome this problem, one can run $k$ independent replications of the system simulation. Assume that run $i$ produces the output data $X_{i 1}, X_{i 2}, \ldots, X_{i n}$. Then the sample means

$$
Y_{i}=\frac{1}{n} \sum_{j=1}^{n} X_{i j}
$$




\section{Alexopoulos and Seila}

are i.i.d. random variables,

$$
\bar{Y}_{k}=\frac{1}{k} \sum_{i=1}^{k} Y_{i}
$$

is also an unbiased estimator of $\mu$, and

$$
\hat{V}_{R}=\frac{1}{k-1} \sum_{i=1}^{k}\left(Y_{i}-\bar{Y}_{k}\right)^{2}
$$

is an unbiased estimator of $\operatorname{Var}\left(\bar{X}_{n}\right)$. If in addition $n$ and $k$ are sufficiently large, an approximate $100(1-\alpha)$ percent confidence interval for $\mu$ is

$$
\bar{Y}_{k} \pm t_{k-1,1-\alpha / 2} \sqrt{\hat{V}_{R} / k}
$$

where $t_{d, \gamma}$ represents the $\gamma$-quantile of the $t$ distribution with $d$ degrees of freedom.

The method of replications can also be used for estimating performance measures other than means. For example, suppose that we want to estimate the $p$-quantile, say $\xi_{p}$, of the average queue size in a single-server queueing system during a fixed time window. We run $k$ independent replications, denote by $Y_{i}$ the average observed queue length during replication $i$, and let $Y_{(1)}<Y_{(2)}<\ldots<Y_{(k)}$ be the order statistics corresponding to the $Y_{i}$ 's. Then a point estimate for $y_{p}$ is

$$
\hat{\xi}_{p}= \begin{cases}Y_{(k p)} & \text { if } k p \text { is an integer } \\ Y_{(\lfloor k p+1\rfloor)} & \text { otherwise }\end{cases}
$$

and a confidence interval for $\xi_{p}$ can be computed as described in Welch (1983, pp. 287-288).

Law and Kelton (1991) review sequential procedures for determining the number of replications required to estimate $\mu$ with a fixed error or precision. Their procedure for obtaining an estimate with a relative error $\left|\bar{Y}_{k}-\mu\right| /|\mu|$ bounded from above by $\gamma$ with probability at least $1-\alpha$ has performed well for initial sample size $k_{0} \geq 10$ and $\gamma \leq 0.15$. A well-known sequential procedure for constructing a $1-\alpha$ confidence interval for $\mu$ with a small absolute error $\left|\bar{Y}_{k}-\mu\right| \leq \beta$ is due to Chow and Robbins (1965) (see also Nadas 1969). Law (1980) observed that the procedure is very sensitive to the value of $\beta$.

\section{INITIALIZATION PROBLEMS FOR STEADY-STATE SIMULATIONS}

One of the hardest problems in steady-state simulations is the removal of the initialization bias. Suppose that $\left\{X_{i}: i \geq 1\right\}$ is a discrete-time output stochastic process from a single run of a steady-state simulation with initial conditions $I$ and assume that, as $n \rightarrow \infty, P\left(X_{n} \leq\right.$ $x \mid I) \rightarrow P(X \leq x)$, where $X$ is the corresponding steadystate random variable. The steady-state mean of the process $\left\{X_{i}\right\}$ is $\mu=\lim _{n \rightarrow \infty} \mathrm{E}\left(X_{n} \mid I\right)$. The problem with the use of the estimator $\bar{X}_{n}$ for a finite $n$ is that $\mathrm{E}\left(\bar{X}_{n} \mid I\right) \neq \mu$.

The most commonly used method for eliminating the bias of $\bar{X}_{n}$ identifies a index $1 \leq l \leq n-1$ and truncates the observations $X_{1}, \ldots, X_{l}$. Then the estimator

$$
\bar{X}_{n, l}=\frac{1}{n-l} \sum_{i=l+1}^{n} X_{i}
$$

is generally less biased than $\bar{X}_{n}$ because the initial conditions primarily affect data at the beginning of a run. Several procedures have been proposed for the detection of a cutoff index $l$ (see Chance and Schruben 1992; Fishman 1996; Gafarian et al. 1978; Goldsman et al. 1994; Kelton 1989; Ockerman 1995; Schruben 1982; Schruben et al. 1983; Welch 1983; Wilson and Pritsker 1978a,b).

The graphical procedure of Welch (1983) is popular due to its simplicity and generality. This method uses $k$ independent replications with the $i$ th replication producing observations $X_{i 1}, X_{i 2}, \ldots, X_{i n}$ and computes the averages

$$
\bar{X}_{j}=\frac{1}{k} \sum_{i=1}^{k} X_{i j}, \quad j=1, \ldots, n .
$$

Then for a given time window $w$, the procedure plots the moving averages

$\bar{X}_{j}(w)= \begin{cases}\frac{1}{2 w+1} \sum_{m=-w}^{w} \bar{X}_{j+m} & w+1 \leq j \leq n-w \\ \frac{1}{2 j-1} \sum_{m=-j+1}^{j-1} \bar{X}_{j+m} & 1 \leq j \leq w\end{cases}$

against $j$. This plot shows the convergence of the mean of the output process to the steady-state mean. If the plot is reasonably smooth, then $l$ is chosen to be the value of $j$ beyond which the sequence of moving averages appears to be constant without a trend. Otherwise, a different time window is chosen and a new plot is drawn. The choice of $w$ may be a difficult problem for congested systems with output time series having autocorrelation functions with very long tails (see Alexopoulos and Seila 1998, Example 7).

\section{STEADY-STATE ANALYSIS}

We focus on estimation methods for the steady-state mean $\mu$ of a discrete-time output process. Analogous methods for analyzing continuous-time output data are described in a variety of texts (Bratley, Fox, and Schrage 1987; Fishman 1978; Law and Kelton 1991). The process $\left\{X_{i}\right\}$ is called strictly stationary if the joint distribution of $X_{i+j_{1}}, X_{i+j_{2}}, \ldots, X_{i+j_{k}}$ is independent of $i$ for all 
indices $j_{1}, j_{2}, \ldots, j_{k}$. If $\mathrm{E}\left(X_{i}\right)=\mu, \operatorname{Var}\left(X_{i}\right)<\infty$ for all $i$, and the $\operatorname{Cov}\left(X_{i}, X_{i+j}\right)$ is independent of $i$, then $\left\{X_{i}\right\}$ is called weakly stationary.

\subsection{The Replication/Deletion Approach}

This approach runs $k$ independent replications, each of length $n$ observations, and uses the method of Welch (1983) to discard the first $l$ observations from each run. One then uses the i.i.d. sample means

$$
Y_{i}=\frac{1}{n-l} \sum_{j=l+1}^{n} X_{i j}
$$

to compute point and interval estimators for the steady-state mean $\mu$ (see section 2). The method is simple and general. The following list contains important observations about $l$, $n$ and $k$. (a) As $l$ increases for fixed $n$, the "systematic" error in each $Y_{i}$ due to the initial conditions decreases. However, the sampling error increases because of the smaller number of observations. (b) As $n$ increases for fixed $l$, the systematic and sampling errors in $Y_{i}$ decrease. (c) The systematic error in the sample means $Y_{i}$ cannot be reduced by increasing the number of replications $k$.

Overall, one must be aware that this approach can require a substantial amount of effort to find a "good" truncation index $l$ as well as a large sample size $n$ and a large number of replications to obtain a confidence interval with the required coverage. It is also potentially wasteful of data as the truncated portion is removed from each replication. The regenerative method (section 4.2) and the batch means method (section 4.3) seek to overcome these disadvantages.

\subsection{The Regenerative Method}

This method was proposed by Crane and Iglehart (1974a,b, 1975) and Fishman $(1973,1974)$. Assume that there are (random) time indices $1 \leq T_{1}<T_{2}<\cdots$ such that the portion $\left\{X_{T_{i}}+j, j \geq 0\right\}$ of the output process has the same distribution for each $i$ and is independent of the portion prior to time $T_{i}$. A process with this property is said to be regenerative, and the portion of the process between two successive regeneration epochs is called a cycle. Let $Y_{i}=\sum_{j=T_{i}}^{T_{i+2}-1} X_{j}$ and $Z_{i}=T_{i+1}-T_{i}$ for $i=1,2, \ldots$ and assume that $\mathrm{E}\left(Z_{i}\right)<\infty$. Then the mean $\mu$ is given by

$$
\mu=\frac{\mathrm{E}\left(Y_{1}\right)}{\mathrm{E}\left(Z_{1}\right)} .
$$

Now suppose that one simulates the process $\left\{X_{i}\right\}$ over $n$ cycles and collects the observations $Y_{1}, \ldots, Y_{n}$ and $Z_{1}, \ldots, Z_{n}$. Then

$$
\hat{\mu}=\frac{\bar{Y}_{n}}{\bar{Z}_{n}}
$$

is a strongly consistent, although typically biased for finite $n$, estimator of $\mu$. Furthermore, confidence intervals for $\mu$ can be constructed by using the random variables $Y_{i}-\mu Z_{i}, i=1, \ldots, n$ and the central limit theorem (see Iglehart 1975).

The regenerative method is often difficult to apply in practice because most simulations have either no identifiable regeneration points or very long cycle lengths. Inventory systems and highly reliable communications systems with repairs are two classes of systems to which this method has successfully been applied.

\subsection{The Batch Means Method}

The method of batch means is frequently used to estimate the steady-state mean $\mu$ or the $\operatorname{Var}\left(\bar{X}_{n}\right)$ (for finite $n$ ) and owes its popularity to its simplicity and effectiveness.

To motivate the method, suppose temporarily that the data $X_{1}, \ldots, X_{n}$ are from a weakly stationary process with $\lim _{n \rightarrow \infty} n \operatorname{Var}\left(\bar{X}_{n}\right)<\infty$. Split the data into $k$ batches, each consisting of $b$ observations. (Assume $n=k b$.) The $i$ th batch consists of the observations $X_{(i-1) b+1}, X_{(i-1) b+2}, \ldots, X_{i b}$, for $i=1,2, \ldots, k$, and the $i$ th batch mean is given by

$$
Y_{i}(b)=\frac{1}{b} \sum_{j=1}^{b} X_{(i-1) b+j} .
$$

For fixed $m$, let $\sigma_{m}^{2}=\operatorname{Var}\left(\bar{X}_{m}\right)$. Since the batch means process $\left\{Y_{i}(b), i \geq 1\right\}$ is also weakly stationary, some algebra yields

$$
\sigma_{n}^{2}=\frac{\sigma_{b}^{2}}{k}\left(1+\frac{n \sigma_{n}^{2}-b \sigma_{b}^{2}}{b \sigma_{b}^{2}}\right) .
$$

As a result, $\sigma_{b}^{2} / k$ approximates $\sigma_{n}^{2}$ with error that diminishes as first $n \rightarrow \infty$ and then $b \rightarrow \infty$ with $b / n \rightarrow 0$. Equivalently, the correlation among the batch means diminishes as $b$ and $n$ approach infinity with $b / n \rightarrow 0$.

To use the last limiting property, one forms the grand batch mean

$$
\bar{X}_{n}=\frac{1}{k} \sum_{i=1}^{k} Y_{i}(b),
$$

estimates $\sigma_{b}^{2}$ by

$$
\hat{V}_{B}(n, k)=\frac{1}{k-1} \sum_{i=1}^{k}\left(Y_{i}(b)-\bar{X}_{n}\right)^{2},
$$

and computes the following approximate $1-\alpha$ confidence interval for $\mu$ :

$$
\bar{X}_{n} \pm t_{k-1,1-\alpha / 2} \sqrt{\hat{V}_{B}(n, k) / k} .
$$




\section{Alexopoulos and Seila}

The main problem with the application of the batch means method in practice is the choice of the batch size $b$. The literature contains several batch selection approaches for fixed sample size; see Conway (1963), Fishman (1978), Law and Carson (1979), Mechanic and McKay (1966), and Schriber and Andrews (1979). Schmeiser (1982) reviews the above procedures and concludes that selecting between 10 and 30 batches should suffice for most simulation experiments. The major drawback of these methods is their inability to yield a consistent variance estimator.

\subsection{Consistent Estimation Batch Means Methods}

Consistent estimation batch means methods assume the existence of a parameter $\sigma_{\infty}^{2}$ (the time-average variance of the process $\left\{X_{i}\right\}$ ) such that a central limit theorem holds

$$
\sqrt{n}\left(\bar{X}_{n}-\mu\right) \stackrel{\mathcal{D}}{\longrightarrow} \sigma_{\infty} N(0,1) \quad \text { as } n \rightarrow \infty
$$

and aim at constructing a consistent estimator for $\sigma_{\infty}^{2}$ and an asymptotically valid confidence interval for $\mu$.

Chien et al. (1997) considered stationary processes and, under quite general moment and sample path conditions, showed that as both $b, k \rightarrow \infty, \operatorname{MSE}\left(b \hat{V}_{k}(b)\right) \rightarrow 0$. Notice that mean squared error consistency differs from consistency.

The limiting result (4) is implied under the following two assumptions, where $\{W(t), t \geq 0\}$ is the standard Brownian motion process (see Resnick 1994, Chapter 6).

Assumption of Weak Approximation (AWA). There exist finite constants $\mu$ and $\sigma_{\infty}>0$ such that

$$
\frac{n\left(\bar{X}_{n}-\mu\right)}{\sigma_{\infty}} \stackrel{\mathcal{D}}{\longrightarrow} W(n) \text { as } n \rightarrow \infty .
$$

Assumption of Strong Approximation (ASA). There exist finite constants $\mu, \sigma_{\infty}>0, \lambda \in(0,1 / 2]$, and a finite random variable $C$ such that, with probability one,

$$
\left|n\left(\bar{X}_{n}-\mu\right)-\sigma_{\infty} W(n)\right| \leq C n^{1 / 2-\lambda} \quad \text { as } n \rightarrow \infty .
$$

The ASA is not restrictive as it holds under relatively weak assumptions for a variety of stochastic processes including Markov chains, regenerative processes and certain queueing systems (see Damerdji 1994). The constant $\lambda$ is closer to $1 / 2$ for processes having little autocorrelation, while it is closer to zero for processes with high autocorrelation.

\subsection{Batching Rules}

Fishman and Yarberry (1997) and Fishman (1996, Chapter 6) presented a thorough discussion of batching rules. Equation (2) suggests that fixing the number of batches and letting the batch size grow as $n \rightarrow \infty$ ensures that $\sigma_{b}^{2} / k \rightarrow \sigma_{n}^{2}$. This motivates the following rule.
The Fixed Number of Batches (FNB) Rule. Fix the number of batches at $k$. For sample size $n$, use batch size $b_{n}=\lfloor n / k\rfloor$.

The FNB rule along with AWA imply that, as $n \rightarrow \infty$, $\bar{X}_{n} \stackrel{\mathcal{P}}{\longrightarrow} \mu$ and

$$
\frac{\bar{X}_{n}-\mu}{\sqrt{\hat{V}_{B}(n, k) / k}} \stackrel{\mathcal{D}}{\longrightarrow} t_{k-1}
$$

(see Glynn and Iglehart 1990). Hence, (3) is an asymptotically valid confidence interval for $\mu$. Unfortunately, the FNB rule has two major limitations: (a) Since $b_{n} \hat{V}_{k}(b)$ is not a consistent estimator of $\sigma_{\infty}^{2}$, the confidence interval (3) tends to be wider than the interval a consistent estimation method would produce. (b) Statistical fluctuations in the halfwidth of the confidence interval (3) do not diminish relative to statistical fluctuation in the sample mean (see Fishman 1996, pp. 544-545).

The limitations of the FNB rule can be removed by simultaneously increasing the batch size and the number of batches. Indeed, assume that ASA holds and consider batch sizes of the form $b_{n}=\left\lfloor n^{\theta}\right\rfloor, \theta \in(1-2 \lambda, 1)$. Then as $n \rightarrow \infty, \bar{X}_{n} \stackrel{a . s .}{\longrightarrow} \mu, b_{n} \hat{V}_{B}\left(n, k_{n}\right) \stackrel{\text { a.s. }}{\longrightarrow} \sigma_{\infty}^{2}$, and

$$
Z_{k_{n}}=\frac{\bar{X}_{n}-\mu}{\sqrt{\hat{V}_{B}\left(n, k_{n}\right) / k_{n}}} \stackrel{\mathcal{D}}{\rightarrow} N(0,1)
$$

(see Damerdji 1994). The last display implies that

$$
\bar{X}_{n} \pm t_{k_{n}-1,1-\alpha / 2} \sqrt{\hat{V}_{B}\left(n, k_{n}\right) / k_{n}}
$$

is an asymptotically valid $1-\alpha$ confidence interval for $\mu$. In particular, the assignment $\theta=1 / 2$ and the SQRT rule below are valid if $1 / 4<\lambda<1 / 2$. Notice that the last inequality is violated by processes having high autocorrelation $(\lambda \approx 0)$.

The Square Root (SQRT) Rule. For sample size $n$, use batch size $b_{n}=\lfloor\sqrt{n}\rfloor$ and number of batches $k_{n}=\lfloor\sqrt{n}\rfloor$.

Under some additional moment conditions, Chien (1989) showed that the convergence of $Z_{k_{n}}$ to the $N(0,1)$ distribution is fastest if both $b_{n}$ and $k_{n}$ grow proportionally to $\sqrt{n}$. Unfortunately, in practice the SQRT rule tends to seriously underestimate the $\operatorname{Var}\left(\bar{X}_{n}\right)$ for fixed $n$.

With the contrasts between the FNB and SQRT rules in mind, Fishman and Yarberry proposed two procedures that dynamically shift between the two rules. Both procedures perform "interim reviews" and compute confidence intervals at times $n_{l} \approx n_{1} 2^{l-1}, l=1,2, \ldots$ using the results of a correlation test for the batch means based on von Neumann's statistic:

$$
C\left(n, k_{n}\right)=1-\frac{\sum_{i=2}^{k}\left(Y_{i}\left(b_{n}\right)-Y_{i-1}\left(b_{n}\right)\right)^{2}}{2 \sum_{i=1}^{k}\left(Y_{i}\left(b_{n}\right)-\bar{X}_{n}\right)^{2}}
$$




\section{Advanced Methods for Simulation Output Analysis}

The LBATCH Procedure. At time $n_{l}$, if the hypothesis test detects autocorrelation between the batch means, the batching for the next review is determined by the FNB rule. If the test fails to detect correlation, all future reviews omit the test and employ the SQRT rule.

The ABATCH Procedure. If at time $n_{l}$ the hypothesis test detects correlation between the batch means, the next review employs the FNB rule. If the test fails to detect correlation, the next review employs the SQRT rule.

Both procedures yield random sequences of batch sizes. Under relatively mild assumptions, these sequences imply convergence results analogous to (5). The respective algorithms require $O(n)$ time and $O\left(\log _{2} n\right)$ space, where $n$ is the desired sample size (see Yarberry 1993 and Alexopoulos et al. 1997). Although like complexities are known for static fixed batch size algorithms, the dynamic setting of the LBATCH and ABATCH rules offers an important additional advantage not present in the static approach. As the analysis evolves with increasing sample path length, it allows a user to assess how well the estimated variance of the sample mean stabilizes. This assessment is essential to gauge the quality of the confidence interval for the sample mean. The LABATCH.2 implementation (described in Fishman 1998a,b) is the only computer package that automatically generates the data for this assessment.

\section{Overlapping Batch Means}

An interesting variation of the traditional batch means method is the method of overlapping batch means (OBM) proposed by Meketon and Schmeiser (1984). For given batch size $b$, this method uses all $n-b+1$ overlapping batches to estimate $\mu$ and $\operatorname{Var}\left(\bar{X}_{n}\right)$. The first batch consists of observations $X_{1}, \ldots, X_{b}$, the second batch consists of $X_{2}, \ldots, X_{b+1}$, etc. The OBM estimator of $\mu$ is

$$
\bar{Y}_{O}=\frac{1}{n-b+1} \sum_{i=1}^{n-b+1} Y_{i}(b)
$$

where

$$
Y_{i}(b)=\frac{1}{b} \sum_{j=i}^{i+b-1} X_{j}, \quad i=1, \ldots, n-b+1
$$

are the respective batch means, and has sample variance

$$
\hat{V}_{O}=\frac{1}{n-b} \sum_{i=1}^{n-b+1}\left(Y_{i}(b)-\bar{Y}_{O}\right)^{2} .
$$

The following are properties of the estimators $\bar{Y}_{O}$ and $\hat{V}_{O}$ : (a) The OBM estimator is a weighted average of nonoverlapping batch means estimators. (b) Asymptotically (as $n, b \rightarrow \infty$ and $b / n \rightarrow 0$ ), the OBM variance estimator $\hat{V}_{O}$ and the non-overlapping batch means variance estimator $\hat{V}_{B} \equiv \hat{V}_{B}(n, k)$ have the same expectation, but $\operatorname{Var}\left(\hat{V}_{O}\right) / \operatorname{Var}\left(\hat{V}_{B}\right) \rightarrow 2 / 3$ (Meketon and Schmeiser 1984). (c) The behavior of $\operatorname{Var}\left(\hat{V}_{O}\right)$ appears to be less sensitive to the choice of the batch size than the behavior of $\operatorname{Var}\left(\hat{V}_{B}\right)$ (Song and Schmeiser 1995, Table 1). (d) If $\left\{X_{i}\right\}$ satisfies ASA and $\left\{b_{n}\right\}$ is a sequence of batches with $b_{n}=\left\lfloor n^{\theta}\right\rfloor$, $\theta \in(1-2 \lambda, 1)$ and $b_{n}^{2} / n \rightarrow 0$ as $n \rightarrow \infty$, then (Damerdji 1994) $b_{n} \hat{V}_{O} \stackrel{a . s .}{\longrightarrow} \sigma_{\infty}^{2}$.

Welch (1987) noted that both traditional batch means and overlapping batch means are special cases of spectral estimation at frequency 0 and, more importantly, suggested that overlapping batch means yield near-optimal variance reduction when one forms sub-batches within each batch and applies the method to the sub-batches. For example, a batch of size 64 is split into 4 sub-batches and the first (overlapping) batch consists of observations $X_{1}, \ldots, X_{64}$, the second consists of observations $X_{17}, \ldots, X_{80}$, etc.

\subsection{The Standardized Time Series Method}

This method was proposed by Schruben (1983). The standardized time series is defined by

$$
T_{n}(t)=\frac{\lfloor n t\rfloor\left(\bar{X}_{n}-\bar{X}_{\lfloor n t\rfloor}\right)}{\sigma_{\infty} \sqrt{n}}, \quad 0 \leq t \leq 1
$$

and, under some mild assumptions (e.g., strict stationarity and $\phi$-mixing),

$$
\left(\sqrt{n}\left(\bar{X}_{n}-\mu\right), \sigma_{\infty} T_{n}\right) \stackrel{\mathcal{D}}{\longrightarrow}\left(\sigma_{\infty} W(1), \sigma_{\infty} B\right),
$$

where $\{B(t): t \geq 0\}$ is the standard Brownian bridge process (see Billingsley 1968). Informally, $\left\{X_{i}\right\}$ is $\phi$ mixing if $X_{i}$ and $X_{i+j}$ are approximately independent for large $j$.

If $A=\int_{0}^{1} \sigma_{\infty} B(t) d t$ is the area under $B$, then the identity

$$
\mathrm{E}\left(A^{2}\right)=\sigma_{\infty}^{2} / 12
$$

implies that $\sigma_{\infty}^{2}$ can be estimated by multiplying an estimator of $\mathrm{E}\left(A^{2}\right)$ by 12 . Suppose that the data $X_{1}, \ldots, X_{n}$ are divided into $k$ (contiguous) batches, each of size $b$. Then for sufficiently large $n$ the random variables

$$
A_{i}=\sum_{j=1}^{b}[(n+1) / 2-j] X_{(i-1) b+j}, \quad i=1, \ldots, k
$$

become approximately i.i.d. normal and an estimator of $\mathrm{E}\left(A^{2}\right)$ is

$$
\hat{\mathrm{E}}\left(A^{2}\right)=\frac{1}{\left(b^{3}-b\right) k} \sum_{i=1}^{k} A_{i}^{2} .
$$




\section{Alexopoulos and Seila}

Hence an (approximate) $100(1-\alpha)$ percent confidence interval for $\mu$ is

$$
\bar{Y}_{k} \pm t_{k, 1-\alpha / 2} \sqrt{\hat{V}_{T} / n}
$$

where

$$
\hat{V}_{T}=12 \hat{\mathrm{E}}\left(A^{2}\right) .
$$

The standardized time series method is easy to implement and has asymptotic advantages over the batch means method (see Goldsman and Schruben 1984). However, in practice it can require prohibitively long runs as noted by Sargent, et al. (1992). Some useful theoretical foundations of the method are given in Glynn and Iglehart (1990). Additional developments, as well as other standardized time series estimators, are contained in Goldsman et al. (1990) and Goldsman and Schruben (1984, 1990). Finally, Damerdji (1994) shows that under ASA in section 4.3, batching sequences with $b_{n}=\left\lfloor n^{\theta}\right\rfloor, \theta \in(1-2 \lambda, 1)$, yield asymptotically consistent estimators for the process variance $\sigma_{\infty}^{2}$.

\subsection{Quantile Estimation}

A variety of methods have been proposed for estimating quantiles of steady-state output processes (see Iglehart 1976; Moore 1980; Seila 1982a,b; Heidelberger and Lewis 1984). All methods estimate the quantile using the sample quantile but differ in the way the variance of the sample quantile is estimated. It should be noted that quantile estimation is a harder problem than the estimation of steady-state means.

\subsection{Multivariate Estimation}

Frequently, the output from a single simulation run is used to estimate several system parameters simultaneously. The estimators of these parameters are typically correlated. As an example, consider the average customer delays at two stations on a path of a queueing network. In general, Bonferroni's inequality can be used to compute a conservative simultaneous confidence coefficient for any set of confidence intervals. Suppose that $D_{i}$ is a $100\left(1-\alpha_{i}\right)$ percent confidence interval for the parameter $\mu_{i}, i=1, \ldots, k$. Bonferroni's inequality states that

$$
P\left(\cap_{i=1}^{k}\left\{\mu_{i} \in D_{i}\right\}\right) \geq 1-\sum_{i=1}^{k} \alpha_{i} .
$$

If the overall simultaneous confidence level must be at leașt $100(1-\alpha)$ percent, then the $\alpha_{i}$ 's can be chosen so that $\sum_{i=1}^{k} \alpha_{i}=\alpha$. Other methods for multivariate estimation of the mean can be found in Charnes (1989, 1990, 1991) and Chen and Seila (1987).

\section{REFERENCES}

Alexopoulos, C., and A. F. Seila. 1998. Output data analysis. In Handbook of Simulation, ed. J. Banks, Chapter 7, New York: John Wiley \& Sons.

Alexopoulos, C., A. F. Seila and G. S. Fishman. 1998. Computational experience with the batch means method. In Proceedings of the 1997 Winter Simulation Conference, 194-201. Institute of Electrical and Electronics Engineers, Piscataway, New Jersey.

Billingsley, P. 1968. Convergence of probability measures, Wiley, New York.

Bratley, P., B. L. Fox, and L. E. Schrage. 1987. A guide to simulation, 2d Ed. Springer-Verlag, New York, New York.

Chance, F., and L. W. Schruben. 1992. Establishing a truncation point in simulation output. Technical Report, School of Operations Research and Industrial Engineering, Cornell University, Ithaca, New York.

Charnes, J. M. 1989. Statistical analysis of multivariate discrete-event simulation output. Ph.D. Thesis, Department of Operations and Management Science, University of Minnesota, Minneapolis, Minnesota.

Charnes, J. M. 1990. Power comparisons for the multivariate batch-means method. In Proceedings of the 1990 Winter Simulation Conference, 281-287. IEEE, Piscataway, New Jersey.

Charnes, J. M. 1991. Multivariate simulation output analysis. In Proceedings of the 1991 Winter Simulation Conference, 187-193. IEEE, Piscataway, New Jersey.

Chen, R. D., and A. F. Seila. 1987. Multivariate inference in stationary simulation using batch means. In Proceedings of the 1987 Winter Simulation Conference, 302-304. IEEE, Piscataway, New Jersey.

Chien, C.-H. 1989. Small sample theory for steady state confidence intervals. Technical Report No. 37, Department of Operations Research, Stanford University, Palo Alto, California.

Chien, C., D. Goldsman, and B. Melamed. 1997. Largesample results for batch means. Management Science 43:1288-1295.

Chow, Y. S., and H. Robbins. 1965. On the asymptotic theory of fixed-width sequential confidence intervals for the mean. Annals of Mathematical Statistics 36:457-462.

Conway, R. W. 1963. Some tactical problems in digital simulation. Management Science 10:47-61.

Crane, M. A., and D. L. Iglehart. 1974a. Simulating stable stochastic systems, I: General multiserver queues. Journal of the ACM 21:103-113.

Crane, M. A., and D. L. Iglehart. 1974b. Simulating stable stochastic systems, II: Markov chains. Journal of the ACM 21:114-123. 


\section{Advanced Methods for Simulation Output Analysis}

Crane, M. A., and D. L. Iglehart. 1975. Simulating stable stochastic systems III: Regenerative processes and discrete-event simulations. Operations Research 23:33-45.

Damerdji, H. 1994. Strong consistency of the variance estimator in steady-state simulation output analysis. Mathematics of Operations Research 19:494-512.

Fishman, G. S. 1973. Statistical analysis for queueing simulations. Management Science 20:363-369.

Fishman, G. S. 1974. Estimation of multiserver queueing simulations. Operations Research 22:72-78.

Fishman, G. S. 1978. Principles of discrete event simulation, New York: John Wiley \& Sons.

Fishman, G. S. 1996. Monte Carlo: Concepts, algorithms, and applications. New York: Springer-Verlag.

Fishman, G. S. 1998a. LABATCH.2 for analyzing sample path data. Technical Report UNC/OR TR-97/04, Department of Operations Research, University of North Carolina, Chapel Hill, North Carolina.

Fishman, G. S. 1998b. LABATCH2: Software for statistical analysis of simulation sample data. In Proceedings of the 1998 Winter Simulation Conference, this volume. Institute of Electrical and Electronics Engineers, Piscataway, New Jersey.

Fishman, G. S., and L. S. Yarberry. 1997. An implementation of the batch means method. INFORMS Journal on Computing 9:296-310.

Gafarian, A.V., C. J. Ancker, and F. Morisaku. 1978. Evaluation of commonly used rules for detecting steady-state in computer simulation. Naval Research Logistics Quarterly 25:511-529.

Glynn, P. W., and D. L. Iglehart. 1990. Simulation analysis using standardized time series. Mathematics of Operations Research 15:1-16.

Goldsman, D., M. Meketon, and L. W. Schruben. 1990. Properties of standardized time series weighted area variance estimators. Management Science 36:602-612.

Goldsman, D., and L. W. Schruben. 1984. Asymptotic properties of some confidence interval estimators for simulation output. Management Science 30:1217-1225.

Goldsman, D., and L. W. Schruben. 1990. New confidence interval estimators using standardized time series. Management Science 36:393-397.

Goldsman, D., L. W. Schruben, and J. J. Swain. 1994. Tests for transient means in simulated time series. Naval Research Logistics 41:171-187.

Heidelberger, P., and P. A. W. Lewis. 1984. Quantile estimation in dependent sequences. Operations Research 32:185-209.

Heidelberger, P., and P. D. Welch. 1981a. A spectral method for confidence interval generation and run length control in simulations. Communications of the ACM 24:233-245.
Iglehart, D. L. 1975. Simulating stable stochastic systems, V: Comparison of ratio estimators. Naval Research Logistics Quarterly 22:553-565.

Iglehart, D. L. 1976. Simulating stable stochastic systems, VI: Quantile estimation. Journal of the ACM 23:347360.

Iglehart, D. L. 1978. The regenerative method for simulation analysis. In Current Trends in Programming Methodology, Vol. III, eds. K. M. Chandy, and K. M. Yeh, 52-71. Prentice-Hall, Englewood Cliffs, New Jersey.

Kelton, W. D. 1989. Random initialization methods in simulation. IIE Transactions 21:355-367.

Law, A. M., and J. S. Carson. 1979. A sequential procedure for determining the length of a steady-state simulation. Operations Research 27:1011-1025.

Law, A. M., and W. D. Kelton. 1991. Simulation modeling and analysis, 2d Ed. McGraw-Hill, New York.

Mechanic, H., and W. McKay. 1966. Confidence intervals for averages of dependent data in simulations II. Technical Report ASDD 17-202, IBM Corporation, Yorktown Heights, New York.

Meketon, M. S., and B. W. Schmeiser. 1984. Overlapping batch means: Something for nothing? In Proceedings of the 1984 Winter Simulation Conference, 227-230. IEEE, Piscataway, New Jersey.

Moore, L. W. 1980. Quantile estimation in regenerative processes. Ph.D. Thesis, Curriculum in Operations Research and Systems Analysis, University of North Carolina, Chapel Hill, North Carolina.

Nadas, A. 1969. An extension of the theorem of Chow and Robbins on sequential confidence intervals for the mean. Annals of Mathematical Statistics 40:667-671.

Ockerman, D. H. 1995. Initialization bias tests for stationary stochastic processes based upon standardized time series techniques. Ph.D. Thesis, School of Industrial and Systems Engineering, Georgia Institute of Technology, Atlanta, Georgia.

Resnick, S. I. 1994. Adventures in stochastic processes. Boston: Birkhaüser.

Sargent, R. G., K. Kang, and D. Goldsman. 1992. An investigation of finite-sample behavior of confidence interval estimators. Operations Research 40:898-913.

Schmeiser, B. W. 1982. Batch size effects in the analysis of simulation output. Operations Research 30:556-568.

Schriber, T. J., and R. W. Andrews. 1979. Interactive analysis of simulation output by the method of batch means. In Proceedings of the 1979 Winter Simulation Conference, 513-525. IEEE, Piscataway, New Jersey.

Schruben, L. W. 1982. Detecting initialization bias in simulation output. Operations Research 30:569-590. 
Schruben, L. W. 1983. Confidence interval estimation using standardized time series. Operations Research 31:1090-1108.

Schruben, L. W., H. Singh, and L. Tierney. 1983. Optimal tests for initialization bias in simulation output. Operations Research 31:1167-1178.

Seila, A. F. 1982a. A batching approach to quantile estimation in regenerative simulations. Management Science 28:573-581.

Seila, A. F. 1982b. Percentile estimation in discrete event simulation. Simulation 39:193-200.

Song, W.-M. T., and B. W. Schmeiser. 1993. Optimal mean-squared-error batch sizes. Management Science 41:110-123.

von Neumann, J. 1941. Distribution of the ratio of the mean square successive difference and the variance. Annals of Mathematical Statistics 12:367-395.

Welch, P. D. 1983. The statistical analysis of simulation results. In The computer performance modeling handbook, ed. S. Lavenberg, 268-328. New York: Academic Press.

Welch, P. D. 1987. On the relationship between batch means, overlapping batch means and spectral estimation, In Proceedings of the 1987 Winter Simulation Conference, 320-323. IEEE, Piscataway, New Jersey.

Wilson, J. R., and A. A. B. Pritsker. 1978a. A survey of research on the simulation startup problem. Simulation 31:55-58.

Wilson, J. R., and A. A. B. Pritsker. 1978b. Evaluation of startup policies in simulation experiments. Simulation 31:79-89.

Yarberry, L. S. 1993. Incorporating a dynamic batch size selection mechanism in a fixed-sample-size batch means procedure. Ph.D. dissertation, Department of Operations Research, University of North Carolina, Chapel Hill, North Carolina.

\section{AUTHOR BIOGRAPHIES}

CHRISTOS ALEXOPOULOS is an Associate Professor in the School of Industrial and Systems Engineering at the Georgia Institute of Technology. He received his Ph.D. in Operations Research from the University of North Carolina at Chapel Hill. His research interests are in the areas of applied probability, statistics, and optimization of stochastic systems. His recent work involves problems related to the optimal design of telecommunications and transportation systems. Dr. Alexopoulos is a member of INFORMS and the INFORMS College of Simulation. He served as a Co-Editor for the Proceedings of the 1995 Winter Simulation Conference.
ANDREW SEILA is an Associate Professor in the Terry College of Business at the University of Georgia, Athens, Georgia. He received his B.S. in Physics and Ph.D. in Operations Research and Systems Analysis, both from the University of North Carolina at Chapel Hill. Prior to joining the faculty of the University of Georgia, he was a Member of Technical Staff at the Bell Laboratories in Holmdel, New Jersey. His research interests include all areas of simulation modeling and analysis, especially output analysis. He has been actively involved in the Winter Simulation Conference since 1977, and served as Program Chair for the 1994 conference. 